\title{
REDIMAT
}

Instructions for authors, subscriptions and further details:

http://redimat.hipatiapress.com

\section{La Base de Orientación en la Resolución de Problemas: "Cuando me Bloqueo o me Equivoco"}

Joana Villalonga ${ }^{1}$ and Jordi Deulofeu ${ }^{1}$

1) Universitat Autònoma de Barcelona

Date of publication: October $24^{\text {th }}, 2017$

Edition period: October 2017-February 2018

To cite this article: Villalonga, J., \& Deulofeu, J. (2017). La base de orientación en la resolución de problemas: "Cuando me bloqueo o me equivoco." REDIMAT, 6(3), 256-282. doi: 10.1783/redimat.2017.2262

To link this article: http://dx.doi.org/10.17583/redimat.2017.2262

\section{PLEASE SCROLL DOWN FOR ARTICLE}

The terms and conditions of use are related to the Open Journal System and to Creative Commons Attribution License (CC-BY). 


\section{La Base de Orientación en la Resolución de Problemas: "Cuando me Bloqueo o me Equivoco"}

Joana Villalonga

Universitat Autònoma de

Barcelona
Jordi Deulofeu

Universitat Autònoma de Barcelona

(Recibido: 14 Septiembre 2016; Aceptado: 06 Octubre 2017;

Publicado: 24 Octubre 2017)

\section{Resumen}

Ante los indicios de que el uso de una adecuada base orientación contribuye en la adquisición de la competencia de resolución de problemas, con el presente artículo se pretende examinar parte de aquellos indicios que contribuyen a dicha afirmación al tiempo que profundizar en su interpretación. Para ello se consideran las resoluciones de un problema matemático utilizando una base de orientación por alumnos de entre 11 y 13 años de edad. El estudio se centra en explorar cómo la dimensión dedicada al atasco, que comprende el común error y el temido bloqueo, se ve reflejada en las resoluciones de los alumnos. El análisis llevado a cabo confirma la importancia de la dimensión dedicada al atasco en la base de orientación y su implicación tanto en el proceso de aprendizaje de los alumnos como en la práctica de los docentes. Entretanto, se obtiene una clasificación de las distintas situaciones de atasco y su vinculación con las nueve dimensiones de la base de orientación utilizada.

Palabras Clave: Atasco, autorregulación, base de orientación, resolución de problemas 
REDIMAT, Vol. 6 No. 3 October 2017 pp. 256-282

\section{The Orienting Base in Problem Solving: "When my Mind Goes Blank or I Make a Mistake"}

Joana Villalonga

Universitat Autònoma de

Barcelona
Jordi Deulofeu

Universitat Autònoma de

Barcelona

(Received: 14 September 2016; Accepted: 06 October 2017;

Published: 24 October 2017)

\section{Abstract}

In light of the use of an appropriate orientation basis contributes to the acquisition of problem solving competency, the aim of the present study is to examine and go more deeply into some of those signs that contribute to this statement. For this purpose, students between 11 and 13 years old solved a mathematics problem using an orientation basis. The study explores how the dimension of the orientation basis responsible for the fact of getting stuck (characterized as doing an error or when the mind goes blank) is reflected on students' solutions. The analysis of the students' solutions reveals that orientation basis addressed to mathematics problem solving must not avoid an explicit dimension comprising the fact of getting stuck. Moreover, it reveals implications for both students and teachers. While doing so, a classification of six different stuck situations and its relationship with the nine dimensions of the orientation basis are obtained.

Keywords: Getting stuck, self-regulation, orientation basis, problem solving 


\section{Villalonga \& Deulofeu - La Base de Orientación}

$\mathrm{E}$

1 presente artículo forma parte de un estudio más amplio centrado en caracterizar propuestas de trabajo que permitan mejorar la adquisición de la competencia de resolución de problemas en la transición de la Educación Primaria a la Educación Secundaria a través de acciones del profesorado relacionadas con el diseño del material (problemas) y su aplicación en el aula de manera que comporten un aprendizaje significativo y competencial.

Un estudio previo (Villalonga y Deulofeu, 2015) dio indicios de cómo el uso de una adecuada base de orientación al resolver un problema puede resultar una buena herramienta, así como una práctica satisfactoria en la adquisición de la competencia en resolución de problemas. En este sentido, se percibió que, si bien es verdad que no hay una receta general para resolver problemas, el uso de una base de orientación apropiada permite al resolutor no experto considerar e indagar entre las herramientas y procedimientos matemáticas de qué dispone, proporcionándole seguridad, control y serenidad, a la vez que ayudarle a organizarse en la tarea que debe afrontar. Por ello, con el tiempo y la práctica, puede mejorar su competencia matemática de resolver problemas.

La base de orientación utilizada enfatiza la necesidad de revisar la tarea que con ella se pretende desarrollar, resolver el problema, con el objetivo de que el resolutor no experto adquiera el control necesario, y aprenda a detectar los puntos dónde se bloquea o comete un error, al mismo tiempo que le permita centrarse en encontrar una alternativa y así, con el tiempo, hacerse con dicha dinámica. El análisis de este proceso mediante evidencias de su puesta en práctica, debería no sólo poder discernir cuáles son las dificultades más comunes en el alumnado y su destreza en reconducirlas, sino también exponer cómo permite a los docentes adecuar sus propuestas y desarrollo de actividades.

Para ello, en este artículo se presentan evidencias de uso de una base de orientación para resolver un problema matemático contextualizado en la numeración y el cálculo por parte de los alumnos de una clase de $6^{\circ}$ de Educación Primaria (11-12 años) y dos de $1^{\circ}$ de Educación Secundaria Obligatoria (12-13 años) con su correspondiente análisis. Con ello, se pretende evidenciar y justificar la importancia del estudio de los errores o momentos de bloqueo en los que los alumnos pueden verse involucrados durante la resolución de un problema. Al tomar consciencia de sus dificultades y con su posterior esfuerzo de rectificación siguiendo la base de 
orientación, los alumnos profundizan en la adquisición de la competencia que se pretende. Los docentes, en tanto, adquieren una herramienta para identificar mejor las carencias de su alumnado y, en consecuencia, de los materiales de trabajo, su gestión en el aula y evaluación, pudiendo así adaptarlos mejor, tanto a nivel de cada alumno como en el conjunto de la clase.

Mencionar aquí que con el término general el/los alumno/s nos referimos indistintamente al alumnado de ambos sexos y que con el término resolutor nos referimos a cualquier persona que pretende resolver un problema. En este sentido, entendemos problema matemático por una pregunta matemática presentada en un contexto determinado para la que no se tiene respuesta inmediata o rutinaria.

\section{Marco de Referencia}

\section{La Resolución de Problemas}

La resolución de problemas es una tarea compleja y difícil de orientar. Diferentes son los estudios que se han realizado para hacerle frente. Si bien los primeros trabajos se centraron en describir los procesos necesarios para la resolución de problemas, los posteriores se han focalizado en la identificación de aquellas singularidades de quiénes resuelven los problemas que contribuyen a su éxito. Los estudios más actuales sitúan la planificación y el monitoraje como factores clave en el éxito de la resolución de problemas, así como la influencia de dimensiones afectivas como las creencias, actitudes y emociones (De Corte et al., 2000a 2000b; Schoenfeld, 2007, 2013).

Un problema matemático es, para nosotros, un tipo de pregunta que requiere, por parte de quien lo resuelve, un proceso de investigación matemática (Niss y Højgaard, 2011). Por ello, se hace imprescindible distinguir entre un problema, cuando uno no sabe cómo resolverlo, y un ejercicio, cuando la tarea se puede resolver de manera familiar o rutinaria mediante la aplicación de técnicas o algoritmos, más o menos complicados, pero automatizados (Schoenfeld, 1983). Un problema matemático debe invitar a la búsqueda $\mathrm{y}$, en su resolución tiene que haber una chispa de 


\section{Villalonga \& Deulofeu - La Base de Orientación}

descubrimiento que permita experimentar el encanto de alcanzar el camino para solucionarlo (Burgués y Serramona, 2013).

En relación a las peculiaridades que definen un buen resolutor de problemas, cuatro cualidades han sido especialmente destacadas: el conocimiento de uno mismo, las estrategias de resolución que uno posee (estrategias heurísticas), el propio monitoreo y la autorregulación (aspecto metacognitivo), y las creencias y experiencias previas de uno mismo ante cualquier aspecto de las matemáticas, en general, y de la resolución de problemas, en particular (Schoenfeld, 2013; De Corte et al., 2000b). En este sentido, cabe destacar obras como las de Mason et al. (1982) en la que se introduce la actividad de razonar a través del papel de un monitor interno como si se tratara de un agente independiente al resolutor que va aconsejando qué se debe hacer en cada momento, pero que, de hecho, se vigila y se realiza las preguntas por sí mismo, desarrollando así una regulación personal del proceso.

\section{La autorregulación}

Resolver un problema, como cualquier otra actividad humana, se puede interpretar como un ejemplo de comportamiento dirigido a un objetivo (Schoenfeld, 2007) que requiere la activación de múltiples conocimientos y estrategias (Sanmartí, 2002). Resolver un problema implica un objetivo orientado a una actividad dinámica (Schoenfeld, 2007) en la cual intervienen diversas etapas, no necesariamente desarrolladas en un orden determinado (Burgués y Serramona, 2013). En este sentido, a pesar de que el conocimiento es la base de todo comportamiento competente, la importancia recae en cómo éste se organiza y se accede a él (Schoenfeld, 2007). Antes de iniciar un proceso es necesario prever los posibles caminos a seguir, sus etapas intermedias, así como sus posibles resultados. Esta previsión permite no sólo escoger el camino a seguir sino también el orden de las acciones necesarias para aplicarlo (Sanmartí, 2007). Por ello, la resolución de un problema recae no sólo en los conocimientos de quién lo resuelve sino también de sus objetivos, orientaciones y toma de decisiones (Schoenfeld, 2007). Luego, las habilidades metacognitivas de los alumnos resultan agentes autorreguladores de su aprendizaje en resolución de problemas. 
Sin embargo, las evidencias muestran que dichas habilidades reguladoras con frecuencia están ausentes, especialmente en los aprendices más débiles (De Corte y Verschaffel, 2003). Una mayoría de alumnos manifiestan gran inseguridad ante la resolución de un problema, y no terminan los procesos iniciados (Sanmartí, 2002). El principal motivo sitúa la causa en la incapacidad de planificar la tarea que se les propone, debido a que realizan las tareas aplicando diferentes maneras de hacer o de razonar, sin suficiente coherencia ni orden, siguiendo procesos algorítmicos vacíos (Sanmartí, 2002). Cuando dedican más tiempo a pensar y a planificar la estructura de la resolución que no en efectuar los cálculos, acostumbran a resolver mejor los problemas (Sanmartí, 2002), lo que responde al perfil de resolutor experto, caracterizado por invertir mayor tiempo en el análisis del problema, con el objetivo de comprender de qué se trata, y en la planificación del proceso de resolución (Schoenfeld, 2007; De Corte et al., 2000b). Además, con esta anticipación de la acción no solo se obtienen mejores resultados, sino que la tarea resulta más gratificante (Sanmartí, 2002). Por ello, es necesario ayudar a los alumnos aprender a planificar, un proceso que implica la generación de sub-objetivos necesarios para resolver problemas complejos (De Corte et al., 2000b).

Otra de las peculiaridades del resolutor experto es su capacidad de reflejar continuamente sobre el estado de su proceso de resolución (Schoenfeld, 2007; De Corte et al., 2000b). Sin embargo, los alumnos raras veces verbalizan cómo plantean o pretenden resolver un problema, (Sanmartí; 2002). Por ello es necesario capacitarles para que utilicen formas de representación con las que describir y argumentar de manera coherente sus actuaciones y objetivos, y así poder actuar e intervenir de manera consecuente (Sanmartí; 2002). El hábito de ir escribiendo es una garantía para recuperar posteriormente el curso de las propias ideas (Mason et al., 1982).

De todo ello se concluye que los alumnos no se convierten de manera automática o espontánea en aprendices autorregulados (De Corte y Verschaffel, 2003). Se trata de un proceso a largo plazo que constituye en sí mismo un importante objetivo de aprendizaje. Por ello debería ser inducido desde una edad temprana (De Corte y Verschaffel, 2003) y velar por la adquisición de la autorregularización. Es decir, que todo alumno, frente a un nuevo problema que exija aplicar conocimientos aprendidos, fuera capaz 


\section{Villalonga \& Deulofeu - La Base de Orientación}

de anticipar y planificar las operaciones necesarias para resolverlo (Sanmartí, 2007). Schoenfeld (2013) asegura que con las correctas directrices los alumnos pueden convertirse en resolutores más eficientes de problemas. Para conseguirlo, como añade el autor, resulta imprescindible disponer de la comprensión de cómo funcionan las cosas (Schoenfeld, 2013). En este sentido, la modelización del profesor es un factor esencial en el logro de la autorregulación e implica un cambio profundo en las creencias y actitudes hacia la enseñanza (De Corte y Verschaffel, 2003). En consecuencia, sólo con la inmersión de los alumnos en un nuevo entorno de aprendizaje, será posible fomentar sus habilidades de autorregulación cognitivas (De Corte et al., 2000b).

\section{La base de orientación}

Con el fin de orientar los alumnos a llevar a cabo una tarea, Sanmartí (2007) presenta las bases de orientación (llamadas también guías de navegación o cartas de estudio). Tal como presenta la autora, una base de orientación es aquel instrumento que resume de manera gráfica y ordenada la acción a realizar, con el fin de promover que los alumnos anticipen y planifiquen por sí mismos las operaciones que deben llevar a cabo para resolver con éxito los diferentes tipos de tareas escolares. Además, a lo largo del proceso de enseñanza se debe promover su construcción de forma que cada alumno elabore la suya propia para que pueda percibirla como una herramienta útil y particular a sus necesidades, más que como una colección ordenada de instrucciones (Sanmartí, 2007).

Concebimos, así, una base de orientación para la resolución de problemas como una secuencia resumida y ordenada de acciones cuidadosamente pensada, fundamentada en los requerimientos, que un experto puede identificar, de la tarea a realizar y en las necesidades del alumnado, que lleva a resolver un problema de manera satisfactoria, convirtiéndose para el aprendiz en una herramienta útil para la planificación y autonomía, así como para la autoevaluación.

Así, toda base de orientación debe desplegar el conocimiento que los aprendices han de interiorizar con su ayuda (Sanmartí, 2002). Luego, las bases de orientación se convierten en agentes reguladores de la capacidad que se pretende lograr con el proceso de enseñanza. En consecuencia, su 
evaluación se convierte en un proceso imprescindible para la mejora del proceso de enseñanza y aprendizaje.

\section{La gestión del atasco}

En su propuesta de cómo atacar un problema matemático de manera eficaz y cómo ir aprendiendo de la experiencia, Mason et al. (1982) hacen continua referencia al hecho de quedarse atascado, como algo inevitable que sucede a cualquiera y que no debe esconderse. A pesar de ello, la realidad indica que se trata de una situación poco comprendida y aceptada en las aulas, especialmente por los resolutores no expertos, pues a menudo es motivo de frustración y de abandono (Mason et al., 1982). De hecho, en la escuela el error, como tradicionalmente se conoce el desacierto o confusión durante el desarrollo de un proceso, se tiende a considerar como algo negativo que los alumnos aprenden rápidamente a ocultar para no ser penalizados (Sanmartí, 2007). Sin embargo, el error es el fundamento del desarrollo cultural de la humanidad. Cada individuo construye sus ideas a partir de sus percepciones e interacciones con otros y para llegar a compartir el conocimiento elaborado deben superarse obstáculos de tipología diversa (Sanmartí, 2007). De hecho, se ha comprobado que los alumnos que tienen éxito en la escuela se caracterizan por su capacidad para identificar y corregir sus errores. Luego, como no todos los estudiantes desarrollan por si mismos esta capacidad, el reto del docente es comprender sus causas, porque sólo ayudándoles a reconocerlas será posible aprenderlos a corregir (Sanmartí, 2007).

La mejor preparación para hacer frente a una situación de atasco es reconocerlo y aceptarlo. Sólo luego será posible reflexionar sobre las ideas y momentos clave que puedan ser el principio de un nuevo y útil enfoque. Para ello, al intentar resolver un problema, conviene tomar especial nota de los caminos que no lleven a ninguna parte, presentando cada línea de pensamiento lo más elaborada y completa como sea posible, expresándola con claridad y lógica (Mason et al., 1982). Así, en cualquier momento de la resolución, será posible comprobar si se ha omitido algo relevante, si se han deslizado errores, o si es necesario dejar el problema por un tiempo, para retomarlo posteriormente sin perder el trabajo realizado. Descubrir un error o algo inadecuado permite retomar cualquier punto de la resolución. El 
objetivo es descubrirlo, reflexionar y buscar alternativas (Mason et al., 1982).

En definitiva, el error es útil (Sanmartí, 2007) y atascarse es un estado honorable y positivo, del que se puede aprender mucho (Mason, et al., 1982). Es un buen indicador de los procesos intelectuales con los que el alumno afronta la realización de una actividad y, por lo tanto, del desarrollo de la misma, pero, para que se pueda detectar, comprender y favorecer su regulación conviene estimular su expresión (Sanmartí, 2007).

\section{Los Agentes Implicados}

El análisis y posterior reflexión que se pretenden con este estudio surgen de las evidencias de uso una base de orientación para resolver un problema matemático. En esta sección se describen los distintos agentes que han intervenido en dicha aplicación.

\section{Los Participantes}

Las evidencias que se presentan provienen del alumnado de tres aulas de tres centros educativos distintos de Barcelona al resolver un problema utilizando una base de orientación. Una de las tres aulas corresponde a un $6^{\circ}$ curso de Educación Primaria (EP), alumnado de 11 y 12 años de edad; y las otras dos con aulas de $1^{\circ}$ de Educación Secundaria Obligatoria (ESO), alumnado de 12 y 13 años.

Tabla 1.

Muestra del estudio

\begin{tabular}{llcccc}
\hline Problema & Grupo & Curso & ${ }^{i}$ AL. & iiPROF. & iiiRRR \\
\hline Problema 1 & NB6A & $6^{\circ}$ EP & 22 & 1 & 21 \\
& SC1A & $1^{\circ}$ ESO & 31 & 1 & 24 \\
& AL1C & $1^{\circ}$ ESO & 23 & 1 & 20 \\
\hline
\end{tabular}

i _PROF.: Número de profesores en el aula. ii AL-Número de alumnos matriculados al

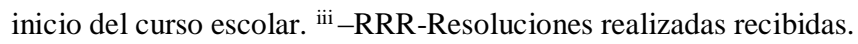


Cabe destacar que, en los tres casos, como es habitual en España, había sólo un profesor en el aula, siendo éste el colaborador con el estudio que presentamos. En la Tabla 1 se detalla la información relativa a cada uno de estos grupos.

No sólo los tres docentes implicados, sino también la realidad de cada una de sus aulas era diferente. NB6A corresponde a un grupo de $6^{\circ}$ de Primaria de un colegio, mientras que SC1A y AL1C son dos grupos de $1^{\circ}$ de la ESO de dos institutos diferentes. El docente del grupo NB6A es maestro, el docente de SC1A es profesor y el del AL1C era maestro que, en su momento, promocionó a trabajar en los primeros cursos de la secundaria. En NB6A trabajan las matemáticas de manera transversal, a través de proyectos, en los que se integra de manera implícita el trabajo con problemas. En SC1A y AL1C trabajan las matemáticas de manera específica, no siguen ningún libro de texto y los alumnos deben tomar apuntes. En el caso de SC1A se facilita un dossier de referencia elaborado por el propio departamento del centro que incluye problemas seleccionados por los profesores. En AL1C se facilitan los problemas que se consideran en cada momento. El docente de AL1C comenta que suele proyectar los enunciados de los problemas, utilizan bastante la calculadora en la resolución de problemas y que suele poner problemas fuera de temario para trabajar de manera voluntaria. SC1A comenta que en su clase no hay una práctica de resolución de problemas individual estricta y que intenta no intervenir mientras los alumnos trabajan los problemas. Destaca mucha falta de autonomía en el proceso de resolución y de aceptación del error. En cualquier de los casos, tanto para docente como alumnos, el uso de una base de orientación para resolver un problema fue una novedad.

\section{El Problema}

Bajo la concepción de que un problema (matemático) debe suponer una propuesta de enfrentamiento con una situación desconocida, planteada mediante un conjunto de datos dentro de un contexto (matemático o no) para la cual no se dispone de una respuesta inmediata si no que requiere reflexionar, tomar decisiones y diseñar estrategias, se propuso un enunciado contextualizado en la misma matemática que conlleva la experimentación numérica. A nuestro juicio, para los alumnos de los cursos implicados la 
situación propuesta promueve una acción de descubrimiento para la cual no tienen una manera directa de resolverlo, pero sí de disponer los conocimientos que, utilizados de manera oportuna, permitan discutirlo y llegar a alguna conclusión.

Tabla 2.

Enunciado del problema

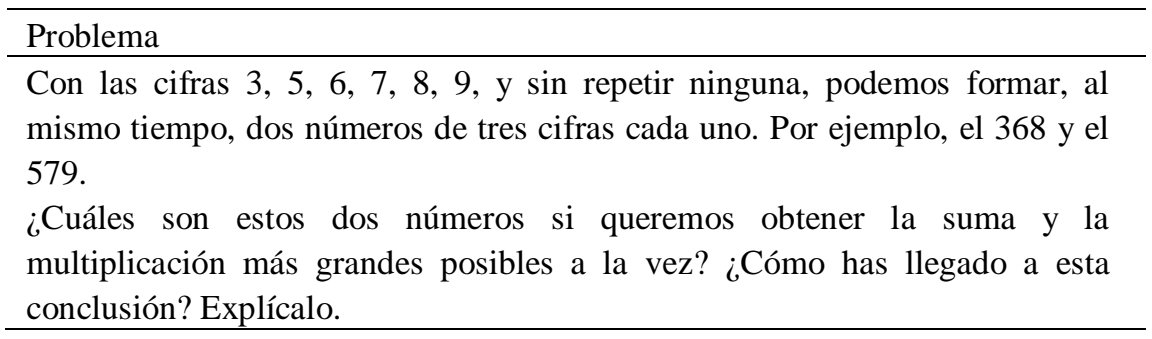

Se consideró oportuno adecuar el problema al ámbito matemático en el cual los alumnos pudieran sentirse más cómodos y para el que tuvieran asimiladas más herramientas. En este sentido, Numeración y el Cálculo se correspondía con el bloque curricular que en primer lugar se trabajaba en todos los cursos implicados y el que, de alguna manera, no dejaba de trabajarse a lo largo del curso escolar en todos los niveles. De ahí que el problema considerado curricularmente encaje en dicho bloque. Por otro lado, y porque los problemas contextualizados en la misma matemática, como los que no, deben también de ser aceptados y suscitar motivación al alumnado, se optó por contextualizar el problema en la misma matemática, a modo de entretenimiento numérico.

\section{La Base de Orientación}

La base de orientación utilizada se basa en las ideas de Pólya (1945), complementadas por las observaciones de De Corte et al. (2000b) y De Corte y Verschaffel (2003), y las propuestas de Mason et al. (1982). Además, se ha construido de acuerdo con los procesos de resolución que, de manera general, se han observado previamente en el alumnado de las edades consideradas. Con todo ello, se han determinado tres dominios de 
actuación para cada uno de los cuales se han identificado tres aspectos característicos y fundamentales, descritos como dimensiones.

En la resolución de un problema, Pólya (1945) determina cuatro fases: entender el problema, concebir un plan de acción, llevar a cabo el plan de acción y volver la mirada atrás. De la primera fase deriva nuestro primer dominio Comprendo el problema que, probablemente debido a su aparente obviedad, a menudo se pasa por alto cuando es un punto clave en la resolución de todo problema (De Corte, 2000b; Mason et al., 1982; Pólya, 1945; Sanmartí, 2007). De la segunda y tercera fase, emerge nuestro segundo dominio Tengo un plan de acción, cuya finalidad abarca el hecho de encontrar una estrategia de resolución, con todo lo que ésta requiera, y así desarrollarla y describirla. La dificultad observada en los alumnos para conceptualmente separar planificación y acción, y bajo las propuestas de Mason et al. (1982), se convino su unión. Finalmente, la visión retrospectiva del proceso, que conlleva la revisión de la tarea realizada y así da nombre al tercer y último dominio de la base de orientación.

La base de orientación se presenta en forma de tabla listada (Tabla 3). Tal como se recalcó a profesores y alumnos (quienes, a su vez, fueron partícipes de su diseño y redacción final), dicha presentación no obliga que los pasos descritos deban mantenerse de forma estricta para resolver el problema. La linealidad establecida, cuyo orden se sustenta por las fuentes usadas y que describen los pasos de un resolutor experto, se convino para posibilitar un orden de aplicación, evitar dispersiones, y al mismo tiempo establecer de manera clara y precisa la relación entre dominios, dimensiones, y ambos. Así mismo, como se puede extraer de ella misma (dimensiones 7 y 8 , especialmente), dicho orden puede quedar alterado y ser reconducido en cualquier momento. De aquí la naturaleza cíclica de resolución de un problema que algunos autores como De Corte et al. (2000a) remarcan.

La descripción de las dimensiones se ha hecho utilizando el presente de indicativo y la primera persona del singular. Con ello se pretende que el resolutor perciba al máximo la proximidad a la persona (ella misma) y al tiempo de resolución (el mismo que su uso). 
Tabla 3.

Base de orientación utilizada

\begin{tabular}{|c|c|}
\hline \multicolumn{2}{|l|}{ Resolución de problemas } \\
\hline Dominios (D) & Dimensiones $(\mathrm{d})$ \\
\hline Comprendo el problema & $\begin{array}{l}{ }^{\mathrm{d} 1} \text { Distingo las preguntas que he de responder y } \\
\text { entiendo todo aquello que se me pide que haga. } \\
{ }^{\mathrm{d} 2} \text { Distingo los datos y me aseguro que los } \\
\text { entiendo. } \\
{ }^{\mathrm{d} 3} \text { Expreso el problema para entenderlo mejor } \\
\text { haciendo un dibujo, esquema, diagrama... (lo } \\
\text { que me parezca más adecuado) y hago pruebas } \\
\text { si me es necesario. }\end{array}$ \\
\hline \multicolumn{2}{|c|}{ Para cada pregunta formulada } \\
\hline $\begin{array}{l}\text { Tengo un plan de } \\
\text { acción }\end{array}$ & $\begin{array}{l}{ }^{\mathrm{d} 4} \text { Pienso alguna estrategia de resolución a partir } \\
\text { de la representación y las pruebas o ejemplos } \\
\text { que he hecho, y trato de aplicarlo. } \\
{ }^{\mathrm{d} 5} \text { Encuentro los datos y los razonamientos y/o } \\
\text { algoritmos que necesito para aplicar la } \\
\text { estrategia. } \\
{ }^{\mathrm{d} 6} \text { Aplico la estrategia y la escribo de manera } \\
\text { que se entienda todo aquello que he pensado. }\end{array}$ \\
\hline Reviso mi tarea & $\begin{array}{l}\text { d7 Si no lo consigo, detecto dónde me bloqueo } \\
\text { o me equivoco y aplico una nueva estrategia } \\
\text { (con todo lo que necesite). } \\
\text { d8 Una vez resuelto, } \\
\text { • investigo si hay otras soluciones y las } \\
\quad \text { encuentro. Si sólo hay una, razono porque } \\
\text { no hay más. } \\
\text { razono si se podría hacer de otras maneras. } \\
\text { d9 Releo lo que he hecho, y me aseguro que lo } \\
\text { explico todo, que respondo de manera } \\
\text { razonada y que se entiende. Relaciono, si hace } \\
\text { falta, con el resto de preguntas y tareas } \\
\text { solicitadas. }\end{array}$ \\
\hline
\end{tabular}


La reflexión que conlleva el presente estudio surge de la primera de las tres últimas dimensiones de la base de orientación Si no lo consigo, detecto dónde me bloqueo o me equivoco y aplico una nueva estrategia (con todo lo que necesite), encargada de ayudar a gestionar los momentos de atasco del resolutor al resolver el problema. Los diferentes matices referidos a un estado de atasco y el interés en que el alumnado se familiarice con ellos, explican por qué en la dimensión el término atascarse se presenta explícitamente como el hecho de equivocarse, de cometer un error, o bien de bloquearse, cuando aparece un momento de confusión. Así mismo, como se ha argumentado anteriormente, cualquier momento de atasco es una situación poco considerada, que requiere de asentamiento, dominio y reflexión de uno mismo y de los procesos que uno lleva a cabo, para la que es imprescindible una mirada atrás, profunda y desde su inicio. De ahí su ubicación en el tercer y último dominio de la base de orientación Reviso mi tarea.

\section{Obtención de los Datos}

La resolución del problema se realizó en una sesión de clase, en el último trimestre del curso 2014-2015. Tanto la base de orientación como el problema utilizados surgen de un proceso de refinamiento, mejor adecuación y consenso con el profesorado implicado después de una primera aplicación durante el 1r Trimestre del mismo curso escolar (Villalonga y Deulofeu, 2015). Con ello, se adecuó la redacción del problema y, por otra, se simplificó la base de orientación para mejorar su comprensión y agilizar su lectura.

Antes de iniciar el proceso de resolución, el profesorado implicado, que previamente se había familiarizado con la herramienta, debía presentar la base de orientación a su alumnado para discutirla con ellos. Era imprescindible asegurarse que tanto el vocabulario y las expresiones utilizadas, como el sentido de la misma fuesen entendidos por los alumnos. En este caso, la base de orientación fue entendida y bien recibida por el alumnado. Para que los alumnos tuvieran acceso a la misma durante la resolución del problema, cada uno de ellos disponía de una copia en papel, para utilizar a su conveniencia. Posteriormente, se debía presentar el problema (Tabla 2) para que cada alumno lo resolviera por escrito y de 


\section{Villalonga \& Deulofeu - La Base de Orientación}

manera individual, siguiendo la base de orientación. También se facilitó a cada alumno una copia impresa del enunciado del problema, donde debían exponer su proceso de resolución. Con el fin de poder acceder al máximo a sus procedimientos y razonamientos, se requería escribir en bolígrafo y no borrar nada de lo escrito. En caso de modificar algo, debían hacer una marca y, si fuera necesario, un pequeño comentario, pero no borrarlo o taparlo. Finalmente, se sugería que se hiciera una revisión por parejas de la resolución del problema, considerando la base de orientación y utilizando un bolígrafo de otro color, preferiblemente, verde.

\section{Resultados}

De las diferentes resoluciones al problema planteado con la base de orientación, han surgido interesantes evidencias en relación a la dimensión dedicada al atasco, caracterizada en nuestro estudio como Si no lo consigo, detecto dónde me bloqueo o me equivoco y aplico una nueva estrategia (con todo lo que necesite). Por la naturaleza del escenario de investigación, en el cual analizamos las resoluciones del alumnado en relación a las herramientas descritas y nos centramos en aquello que concierne a la dimensión detallada, hemos optado por una metodología de análisis cualitativo de las explicaciones redactadas por el alumnado. En este sentido, y siendo fieles a la dinámica más usual en las tres aulas participantes en las que el profesorado de matemáticas percibe mayoritariamente el trabajo de sus alumnos a través de sus escritos, los datos analizados proceden directamente y exclusivamente de aquello que cada alumno escribió en el/los documento(s) dónde desarrollaron su resolución y en el que, posteriormente, un compañero pudo añadir las conclusiones extraídas de la puesta en común.

La finalidad del presente estudio es doble. Por un lado, como acción de investigación, aportar evidencias y discusión significativas a la comunidad investigadora en relación al proceso de enseñanza y aprendizaje de las matemáticas, concretamente, en la adquisición de la competencia en resolución de problemas. Por otro, compartir las herramientas diseñadas para el estudio y su correspondiente análisis con la comunidad educativa, con el propósito que puedan ser adecuadas y utilizadas en la práctica docente habitual. 
Tabla 4.

Situaciones de atasco identificadas

Falta de comprensión

Representaciones

inadecuadas

Estrategia inadecuada

Datos o razonamientos inapropiados

Errores de aplicación

Explicaciones imprecisas
Percatarse de no entender la situación descrita del problema, alguna de sus partes o de los datos que presenta.

Advertir que la representación (expresión o pruebas) realizada de la situación descrita del problema no se corresponde con lo expuesto en dicha situación.

Notar que la estrategia utilizada no es adecuada para la finalidad que se pretende resolver.

Apreciar que se consideran datos o razonamientos no adecuados para aplicar una estrategia.

Reparar que se cometen errores de aplicación.

Percibir que la exposición de las descripciones (explicaciones, conclusiones...) son confusas, impropias o que contienen partes incorrectas o inadecuadas, ya sean de lengua (expresión escrita) o de carácter matemático.

Para dar respuesta a ello, se ejemplifican con partes de las mismas resoluciones, los diferentes tipos de evidencias de atasco (bloqueo o error) encontrados. Concretamente, se han buscado aquellos fragmentos de las resoluciones donde se ha observado que el resolutor debió parar puntualmente su desarrollo, ya sea debido a un momento de confusión (situación de bloqueo) o por haber realizado algo de manera incorrecta 
(error). En definitiva, se ha tratado de discernir cómo actuaron los resolutores en encontrarse ante una situación de atasco, teniendo en cuenta que disponían de la ayuda de la base de orientación. De las sugerencias de Mason et al. (1982) y de las observaciones de Sanmartí (2007), podemos avanzar que el hecho de que el estudiante evidencie que se da cuenta de un estado de atasco da sentido a la dimensión estudiada y, con ello, al uso y aceptación de la base de orientación. Una vez reconocido el bloqueo o el error, la atención recae en cómo se actúa ante ello. En este sentido, el objetivo final persigue que, con la base de orientación, el alumno no sólo sea capaz de detectar una situación de atasco, sino de esforzarse en reconducirlo de manera satisfactoria.

De acuerdo con lo expuesto, identificamos las seis situaciones que se exponen en la Tabla 4.

Para describir los seis tipos de atasco identificados, presentamos y analizamos aquellos fragmentos de las distintas resoluciones más representativos para cada tipo definido. Por cuestiones de espacio, nos limitamos a comentar no más de dos ejemplos por cada tipo.

\section{Falta de Comprensión}

En cada una de estas dos resoluciones, la evidencia más clara de que la situación descrita por el enunciado no es entendida inicialmente, es que ambos alumnos lo manifiestan por escrito. En el primer caso (Figura 1) el alumno hace el esfuerzo de describir qué le conduce al bloqueo. Exponer su confusión y pautar lo que cree que entiende le ayuda a enfocar correctamente el problema.

En el segundo (Figura 2), en lugar de describir el motivo del bloqueo, propone inicialmente una operación combinada de suma y multiplicación con tres números de una cifra (de las seis dadas) cada uno, y se percata que no concuerda con lo descrito en el enunciado; esto le lleva a rectificar y concluir que las cifras deben ser unidas para formar números de tres cifras y cumplir las condiciones requeridas en dos operaciones distintas. 


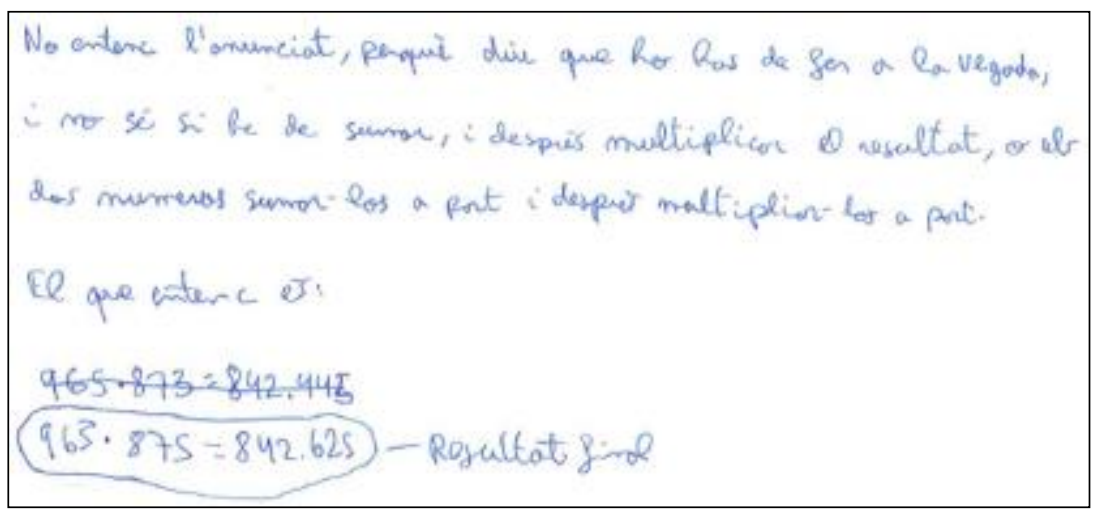

Figura 1. Parte de la resolución SC1A22 ${ }^{1}$

Con ello se evidencia que el hecho de expresar por escrito un estado confusión, y además describir los motivos de ello, o bien de revisar la manera en qué se manipulan los datos del problema, son claves para poder, primero, detectar, aceptar y entender el estado de atasco, y a continuación poder proseguir con la resolución del problema.

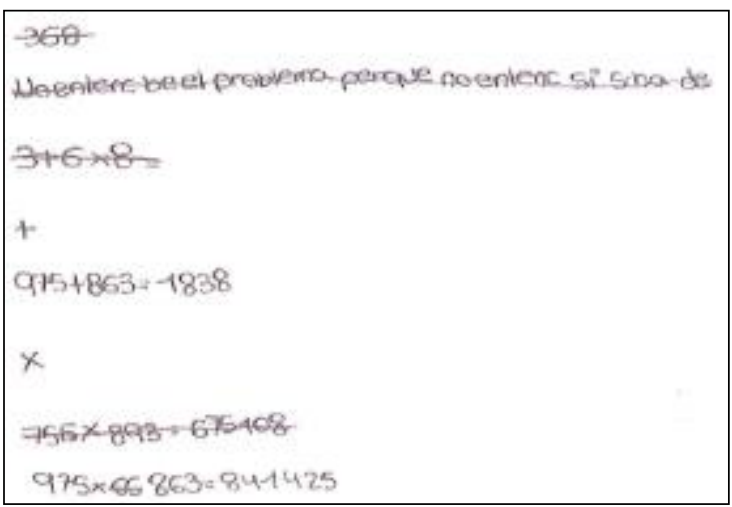

Figura 2. Parte de la resolución SC1A05² 


\section{Representaciones Inadecuadas}

En este fragmento de resolución vemos como antes de expresar una suma y una multiplicación con números de tres cifras distintas, se intenta experimentar con la suma de dos números, uno de tres cifras, pero el otro de dos cifras, con dos de ellas (8 y 5) repetidas en los dos números. Decimos intenta porque en estas experimentaciones iniciales el alumno no anota el resultado de la operación indicada, lo que nos lleva pensar que ya durante la representación tenía sus dudas sobre cómo representar lo expuesto el problema. El hecho de ir anotando distintas interpretaciones del problema le permitieron percatarse de que éstas no concordaban con lo expuesto realmente en él. En este sentido es imprescindible que cada nueva experimentación sea visible para evitar recaer en el error cometido.

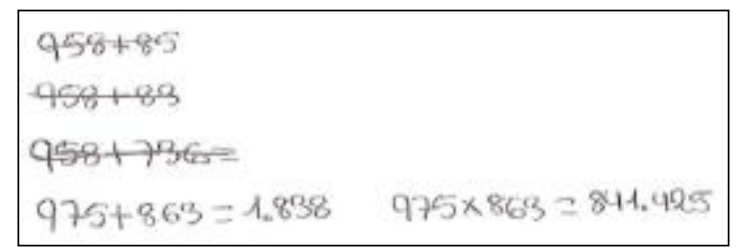

Figura 3. Parte de la resolución SC1A04

\section{Estrategia Inadecuada}

El primer fragmento (Figura 4) evidencia la anulación de una primera estrategia de resolución, mientras que el segundo (Figura 5), que corresponde al mismo alumno, presenta una estrategia alternativa a la primera que, ahora si, es correcta. Si bien la representación del problema está encaminada desde un principio (con los dos números escritos antes de explicitar su primera estrategia da cuenta de que necesita dos números de tres cifras con ninguna de ellas repetida), el alumno se percata de que la estrategia descrita inicialmente no proporciona lo que está buscando, pues los números anotados 987 y 653, son dos números de tres cifras no repetidas, pero no son los que dan la suma y producto máximo. De ahí la modificación de su estrategia inicial a una segunda en la que propone que los dos números de tres cifras más grandes posibles serán los obtenidos al distribuir los dos valores mayores en las centenas, los segundos mayores en 
las decenas y los últimos en las unidades. Esta estrategia se encamina hacia la obtención de la suma y el producto máximo.

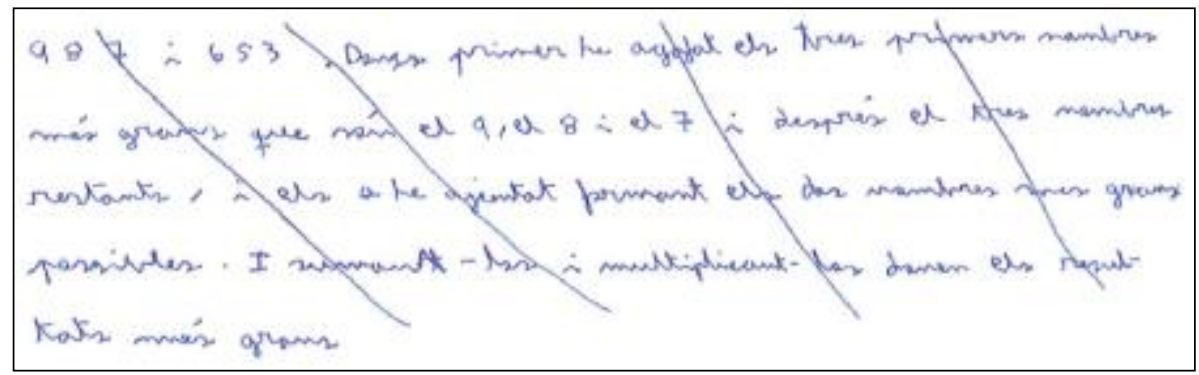

Figura 4. Parte 1 de la resolución $\mathrm{SC} 1 \mathrm{~A} 14^{3}$

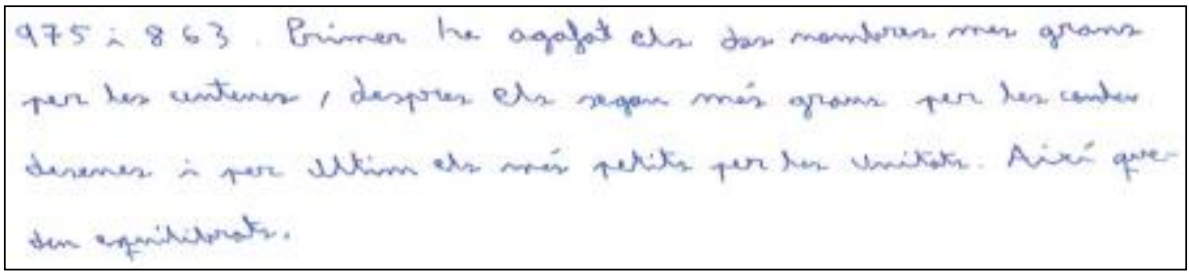

Figura 5. Parte 2 de la resolución $\mathrm{SC} 1 \mathrm{~A} 14^{4}$

Constatamos, pues, que explicitar la estrategia a utilizar, permite volver a ella y no sólo detectar la parte equivocada del razonamiento, sino también rehacerla sin repetir el mismo error o abandonar el intento.

\section{Datos o Razonamientos Inapropiados}

No siempre todos los datos aparentes son relevantes para resolver un problema. Hay que saber descifrarlos y utilizarlos de manera conveniente. Así lo percatan los dos ejemplos aquí presentados. Observamos cómo, en ambos casos, el alumno en cuestión rectifica la selección de unos datos que no son relevantes para la tarea que se disponen a desarrollar. En el primer ejemplo (Figura 6), el resolutor explicita que debe trabajar con 6 cifras. Este 
dato, a pesar que le puede ayudar a comprobar que tiene ese número de cifras qué manipular, no le proporciona información significativa para distribuir las 6 cifras en 2 números de 3 cifras cada uno, y menos para determinar la suma y el producto de mayor valor. De ahí se entiende que acabe tachando dicha información. En el segundo ejemplo (Figura7), vemos un alumno que enfatiza aquellos datos del enunciado que cree relevantes para la resolución subrayándolos en el mismo enunciado. En un primer intento, selecciona los dos números que se habían dado como ejemplo, se da cuenta de que dichos números no satisfacen las condiciones necesarias para resolver el problema y acaba descartándolos. En este sentido, se confirma la necesidad de recordar que no sólo hay que identificar y entender los datos, en general, sino también saber concretar los realmente útiles.

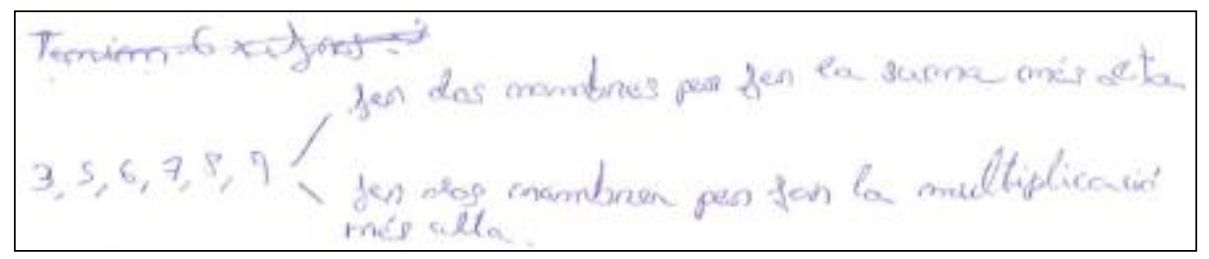

Figura 6. Parte de la resolución $\mathrm{SC} 1 \mathrm{~A} 13^{5}$

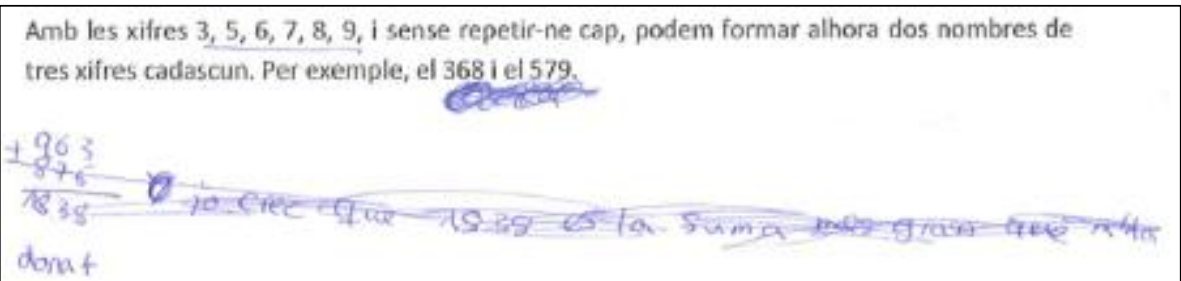

Figura 7. Parte de la resolución SC1A30 


\section{Errores de Aplicación}

Un ejemplo de error de aplicación se observa en esta realización errónea de un cálculo. Por ello conviene recordar la necesidad de revisar las operaciones y la manipulación de los datos en general. Con la revisión e interpretación de los mismos puedan detectarse y corregirse.

$$
963 \times 875=849.625
$$

Figura 8. Parte de la resolución AL1C16

\section{Explicaciones Imprecisas}

\section{Precisión de las explicaciones y argumentaciones}

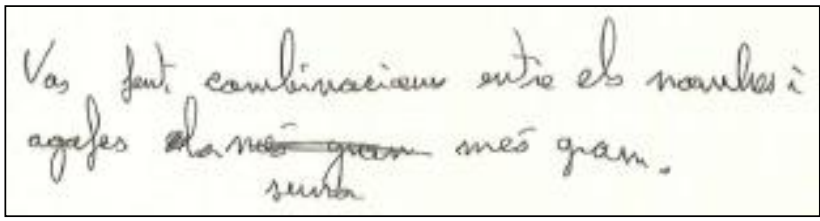

Figura 9. Parte de la resolución AL1C05 ${ }^{6}$

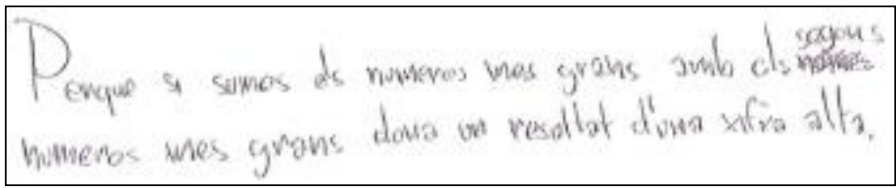

Figura 10. Parte de la resolución NB6A097

\section{Términos y símbolos matemáticos}

Al describir un texto son diversas las imprecisiones que pueden surgir. No es de extrañar, pues, que éstas aparezcan también en la redacción de ideas, razonamientos, o conclusiones sobre un problema. En este sentido, los 
ejemplos expuestos desvelan correcciones en dos sentidos. Por un lado, en la concreción de lo que textualmente se quiere explicar $\mathrm{y}$, por tanto, relacionados con aspectos de la lengua en general, como muestran las Figuras 9 y 10, donde se obvian o anticipan palabras que son clave para la explicación. Por otro, de contexto matemático, ya sea en la precisión de palabras clave, como se ve en la Figura 11, donde inicialmente se utiliza el término decenas en lugar de centenas, o bien al utilizar los símbolos adecuados, como se puede apreciar en las Figura 12, dónde, a pesar de multiplicar, previamente se había indicado una suma. En este sentido, la revisión de los escritos conlleva un asentamiento, adecuación e interpretación del lenguaje, matemático o no, utilizado.

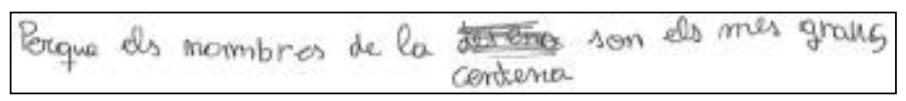

Figura 11. Parte de la resolución NB6A018

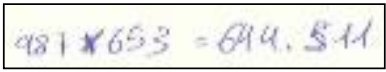

Figura 12. Resolución AL1C19

\section{Discusión y Conclusiones}

Ante las evidencias de que el uso de una adecuada base de orientación para resolver un problema contribuye positivamente en la adquisición de la competencia de resolución de problemas (Villalonga y Deulofeu, 2015), el presente artículo ha pretendido examinar parte de aquellos indicios que contribuyen a tal efecto al tiempo que profundizar en su interpretación.

Para ello, nos hemos centrado en el análisis de una de las dimensiones de la base de orientación, la dedicada a la revisión de un posible atasco: Si no lo consigo, detecto dónde me bloqueo o me equivoco y aplico una nueva estrategia (con todo lo que necesite). De acuerdo con Mason et al. (1982) y Sanmartí (2007), las situaciones de desacierto o confusión se conciben, en general, como algo negativo que se aprenden a ocultar. Siendo éstos un 
punto clave en cualquier proceso de aprendizaje, y más especialmente en la adquisición de la competencia en resolución de problemas, resulta necesario identificarlos y reconocerlos. En este sentido hemos percibido como en la mayoría de los casos analizados, el hecho de utilizar la base de orientación durante la resolución del problema ha permitido a los alumnos identificar la situación personal de atasco en qué se encontraban, al tiempo que eran capaces de revisar su estado, propiciando la búsqueda de una alternativa que, de manera generalizada, ha supuesto una resolución satisfactoria del problema.

De manera paralela, las evidencias obtenidas confirman la necesidad de que cualquier idea o acción que conlleva resolver un problema debe ser escrita y, en ningún caso, eliminada del discurso de la resolución. De no hacerlo se complica la recuperación de cualquier tipo de atasco, entenderlo y, más aún, tener opción a rectificarlo. En este sentido, las evidencias encontradas muestran como, ante cualquier atasco, el hecho de evidenciar el conflicto emergente ha dado pie a su posible rectificación por parte del mismo resolutor, confirmando así que éste ha reconocido y aceptado su puntual estado. Y, de manera consecuente, que cualquier lector de la resolución pueda también dar cuenta de ello.

Con ello desvelamos la necesidad de que, hasta que los alumnos así lo interioricen, la base de orientación destinada a guiar la resolución de un problema, debe contemplar una dimensión específica para la revisión del tradicional error o del temido bloqueo, al tiempo que promover que los alumnos dejen por escrito todas sus ideas y propuestas, sin borrar los intentos que consideren inoportunos. Sólo así será posible una posterior lectura de la resolución, y consecuentemente poder interpretar y entender los procedimientos y decisiones llevados a cabo. Será entonces cuando se podrán identificar y reconocer situaciones de dificultad. Esta dinámica es la que, finalmente, permitirá al alumno que resuelve el problema con la base de orientación, no sólo identificar y reconocer situaciones de atasco, sino encontrar una alternativa, generalmente satisfactoria, en lugar de abandonar la resolución del problema. Al mismo tiempo, esta forma de trabajo permite al docente, identificar y (re)conocer con más profundidad las carencias y necesidades de cada alumno y actuar de manera consecuente.

El análisis de los diferentes momentos de error o bloqueo, ha conllevado el reconocimiento de seis situaciones distintas de atasco (Tabla 4) en las 


\section{Villalonga \& Deulofeu - La Base de Orientación}

que los alumnos, como resolutores de los problemas, se pueden ver involucrados. De ello surgen correspondencias ineludibles entre los seis tipos de atasco descritos (a, b, c, d, e, f en Tabla 4) y el conjunto de dominios y de dimensiones que conforman la base de orientación (ver Figura 13). Si bien una primera lectura evidenció estrechos vínculos entre cada uno de los tipos de atasco con uno de los tres dominios de la base de orientación, una segunda lectura permitió precisar su relación con cada una de las dimensiones de la base de orientación. En este sentido, observamos un estrecho vínculo entre los dos primeros tipos de atasco ( $\mathrm{a}$ y b) con el dominio dedicado al entender el problema; los tres siguientes tipos de atasco (c, d y e) con el dominio dedicado al plan de acción y; el sexto tipo de atasco (f) con el dominio dedicado a la revisión. Al profundizar, observamos como el primer tipo de atasco (a) afecta las dos primeras dimensiones de la base de orientación, y el segundo (b) la tercera dimensión. Los tipos de atasco c), d) y e) afectan directamente las dimensiones 4, 5 y 6 , respectivamente, mientras que el atasco responsable de explicaciones se vincula con la novena dimensión.

Esta correspondencia, descrita por la Figura 13, se convierte en una herramienta que contribuye a entender y a clasificar las dificultades emergentes al resolver un problema y, en consecuencia, guiar la adaptación del material de trabajo (los problemas, la base de orientación y su puesta en práctica en el aula) según la tipología de atasco identificado en cada uno de los alumnos.

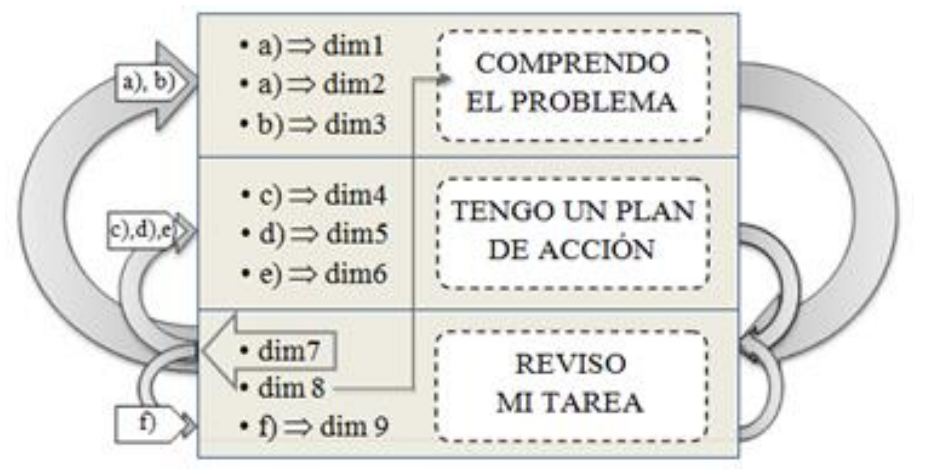

Figura 13. Relación entre las situaciones de atasco identificadas y los dominios y las dimensiones de la base de orientación 
En este sentido, la identificación de las seis situaciones de atasco (Tabla 4) se convierte en el punto de partida para posibles amplificaciones de la base de orientación considerada, especialmente para profundizar el reconocimiento de la naturaleza y el enfrentamiento a los posibles obstáculos. En otras palabras, la clasificación obtenida se convierte en sí misma una particular base de orientación dedicada a guiar y organizar específicamente el enfrentamiento a las situaciones de atasco cuando aún no se ha interiorizado esta dimensión específica dedicada a los obstáculos, proporcionando así completitud a la base de orientación inicial.

Por otro lado, dichas relaciones confirman la naturaleza cíclica del proceso de resolver un problema (De Corte et al., 2000a), característica no siempre evidente en la comunidad escolar. De hecho, a nuestro modo de ver, el mayor grado de esta articulación recae justo en la detección de un estado cualquiera de atasco. Cada tipo de obstáculo requiere una detección y revisión particular y, por tanto, una rectificación o corrección muy específica del proceso identificado. Se confirma así que la resolución un problema es una dinámica compleja, en ningún caso lineal, que requiere de tiempo, reflexión y dedicación.

Concluimos con la necesidad de promover la revisión del atasco en toda base de orientación, ya que, a través de una adecuada reflexión, permite al profesorado el continuo acompañamiento de sus alumnos en el proceso de aprendizaje para la adquisición de la competencia de resolución de problemas. Si bien con la base de orientación se promueve que el alumno deje por escrito una situación de atasco, también la lectura de cómo éste organiza y usa los datos y expone sus desarrollos, da al docente una información muy valiosa para poder evaluar y, en consecuencia, (re)diseñar y (re)adaptar el material de trabajo, tanto los problemas como la base de orientación a utilizar, realzando así las carencias identificadas, de acuerdo con Sanmartí (2007). Dos herramientas para tal efecto, surgidas del artículo, son: la clasificación de situaciones de atasco (Tabla 4), y los vínculos constatados entre el hecho de la revisión del error y las dimensiones establecidas en la base de orientación (Figura 2). 


\section{Villalonga \& Deulofeu - La Base de Orientación}

\section{Notas}

${ }^{1}$ No entiendo el enunciado, porque dice que lo tienes que hacer a la vez, y no sé si se tiene que sumar, y después multiplicar el resultado, o los dos números sumarlos a parte y después multiplicarlos a parte. Lo que entiendo es: $965.873=842.445963 \cdot 875=842.625$ - Resultado final $(\mathrm{NdT})$

${ }^{2}$ No entiendo el problema porque no entieno si se tiene que (NdT)

3987 y 653 . Pues primero he cogido los tres primeros números más grandes que son el 9 , el 8 y el 7 y después los tres números restantes, y los he juntado formando los dos números más grandes posibles. Y sumándolos y multiplicándolos dan los resultados más grandes $(\mathrm{NdT})$

${ }^{4} 975$ i 863. Primero he seleccionado los dos números más grandes para las centenas, después los segundos más grandes para las cien decenas y por último los más pequeños para las unidades. Así quedan equilibrados (NdT)

${ }^{5}$ Tenemes 6 eifras $(\mathrm{NdT})$

${ }^{6}$ Vas haciendo combinaciones entre los números y coges el más grande la suma más grande $(\mathrm{NdT})$

${ }^{7}$ Porque si sumas los números más grandes con los números segundos númeres más grandes da un resultado de una cifra alta $(\mathrm{NdT})$

${ }^{8}$ Porque los números de la decena centena son los más grandes $(\mathrm{NdT})$

\section{Referencias}

Burgués, C., \& Sarramona, J. (Eds.). (2013). Competències bàsiques de l'àmbit matemàtic: A favor de l'èxit escolar. Identificació $i$ desplegament a l'educació secundària obligatòria. Direcció General ESO i Batxillerat. Departament d'Ensenyament. Generalitat de Catalunya. Consultado en: http://ensenyament.gencat.cat/web/.content/ home/departament/publicacions/colleccions/competenciesbasiques/eso/eso-matematic.pdf [marzo 2016]

De Corte, E., Verschaffel, L., \& Greer, B. (2000a). Connecting mathematics problem solving to the real world. In A. Rogerson (Ed.), Proceedings of the International Conference on Mathematics Education into the 21st Century: Mathematics for living (pp. 66-73). Amman, Jordan: The Hong Kong Institute of Education. De Corte, E., Verschaffel, L., \& Op't Eynde, P. (2000b). Self-regulation: A characteristic and a goal of mathematics learning. In M. Boekaerts, P. Pintrich \& M. Zeidner (Eds.), Handbook of self-regulation (pp. 687726). San Diego: Academic Press. 
De Corte, E., \& Verschaffel, L. (2003). El desarrollo de habilidades de autorregulación en la solución de problemas matemáticos.

Pensamiento Educativo, 32, 286-305.

Mason, J., Burton, L., \& Stacey, K. (1982). Thinking mathematically.

Wokingham: Addison-Wesley.

Niss, M. A., \& Højgaard, T. (Eds.) (2011). Competencies and Mathematical Learning: Ideas and inspiration for the development of mathematics teaching and learning in Denmark. Roskilde: Roskilde Universitet. (IMFUFAtekst: i, om og med matematik og fysik; No. 485). Pólya, G. (1945). How to solve it. Princeton: Princeton University Press. Sanmartí, N. (2002). Didáctica de las ciencias en la educación secundaria obligatoria. Barcelona, España: Síntesis Educación.

Sanmartí, N. (2007). Evaluar para aprender: 10 ideas clave. Barcelona, España: GRAÓ.

Schoenfeld, A. H., (1983). The wild, wild, wild, wild, wild world of problem solving (A review of sorts). For the Learning of Mathematics, 3(3), 40-47.

Schoenfeld, A. H. (2007). What is mathematical proficiency and how can it be assessed? Assessing Mathematical Proficiency. MSRI

Publications, 53, 59-73.

Schoenfeld, A. H. (2013). Reflections on problem solving theory and practice. The Mathematics Enthusiast (TME), 10(1y2), 9-34.

Villalonga, J., \& Deulofeu, J. (2015). La base de orientación en la resolución de problemas. En FESPM, SEMRM (Eds.) Actas JAEM 2015. 17 Jornadas para el aprendizaje y la enseñanza de las matemáticas (pp.36, n.68). Cartagena, España: Pedro Ángel Sánchez Martínez, S.L.U. 
Villalonga es estudiante de dotorado en el Departamento de Didáctica de las Matemáticas y de las ciencias Experimentales, Universitat Autònoma de Barcelona, España.

Jordi Deulofeu es profesor titular en el Departamento de Didáctica de las Matemáticas y de las ciencias Experimentales, de la Universitat Autònoma de Barcelona, España.

Dirección de Contacto: La correspondencia directa sobre este artículo se debe enviar al autor. Dirección Postal: Edifici G5, Campus de la UAB, 08193 Bellaterra (Cerdanyola del Vallés). Spain. Email: 